\title{
Vertex Operators and Soliton Solutions of Affine Toda Model with $U(2)$ Symmetry
}

\author{
I. Cabrera-Carnero, J.F. Gomes, G.M. Sotkov and A.H. Zimerman \\ Instituto de Física Teórica - IFT/UNESP \\ Rua Pamplona 145 \\ 01405-900, São Paulo - SP, Brazil
}

\begin{abstract}
The symmetry structure of non-abelian affine Toda model based on the coset $S L(3) / S L(2) \otimes$ $U(1)$ is studied. It is shown that the model possess non-abelian Noether symmetry closing into a $q$-deformed $S L(2) \otimes U(1)$ algebra. Specific two vertex soliton solutions are constructed.
\end{abstract}




\section{Introduction}

The family of 2-D relativistic integrable models (IM) known as affine Toda field theories (ATFT) have been intensively studied due to their relation to certain deformations of 2-d conformal field theories (CFT) [1], [2], [3], [4] as well as due to their rich soliton spectrum [5], [6], [7]. Certain integrable perturbations of $S U(N)$-WZW models and their gauged versions related to ATFT's have also important applications in the condensed matter problems, for example in the description of Heisenberg antiferromagnetic spin chains and ladders [8].

According to their symmetries we distinguish two classes of ATFT's: abelian (A) and nonabelian (NA) ones. The main feature of the non-abelian ATFT's is that they manifest local or global Noether symmetries (say, $U(1)^{\otimes l}, S L(2) \otimes U(1)$, etc ), while the abelian ones do not possess any other symmetries except the discrete $Z_{n}$ in the case of imaginary coupling. As a consequence the NA-ATFT's admit topological solitons carrying certain Noether charges as well. Examples of electrically charged $(U(1)$ or $U(1) \otimes U(1))$ topological solitons have been constructed in refs. [9], [10], [11], [12]. As it is well known [13], such finite energy classical solutions play a crucial role in the semiclassical quantization as well as for establishing their strong-coupling particle spectra [14]. The exact quantum S-matrices of certain T-self dual NA Toda models [15] with $U(1)$ symmetry have been derived in ref. [16].

Although the general theory for constructing of Non-Abelian Affine Toda theories is well developed in terms of graded affine algebras (see for instance [17], [18] and refs. therein), its application to specific NA-ATFT possessing non-abelian symmetries gives rise to certain unexpected and interesting structures. One of them is related to the existence of pairs of T-dual NA-ATFT models described in ref. [19]. In the present paper we will discuss a $q$-deformed algebraic structure that appears in the simplest integrable model of this type, based on the coset $S L(3) / S L(2) \otimes U(1)$, constructed in Sect. 2 (see also Sect. 6 of [11]). In Sect. 3 we show that it is invariant under specific non-local and non-abelian transformations and the corresponding Noether charges close a $q$-deformed $S L(2, R) \otimes U(1)$ Poisson bracket algebra. As a consequence its soliton solutions carry both $U(1)$ and isospin charges. The goal of this paper is to explicitly construct these 1-soliton solutions and to investigate their internal non-abelian symmetries. The two-vertex soliton solutions obtained in Sects. 4 and 5 by the dressing method represent a specific subclass of 1-solitons whose spectrum depends on one real parameter only. Their masses and charges are derived in Sect. 5.

\section{Affine Toda Models with Non-Abelian Symmetries}

\subsection{The model in the group $G_{0}$}

As it is well known [18], the generic NA Toda models are classified according to a $\mathcal{G}_{0} \subset \mathcal{G}$ embedding induced by the grading operator $Q$ decomposing an finite or infinite dimensional Lie algebra $\mathcal{G}=\oplus_{i} \mathcal{G}_{i}$ where $\left[Q, \mathcal{G}_{i}\right]=i \mathcal{G}_{i}$ and $\left[\mathcal{G}_{i}, \mathcal{G}_{j}\right] \subset \mathcal{G}_{i+j}$. A group element $g$ can then be written in terms of the Gauss decomposition as

$$
g=N B M
$$


where $N=\exp \mathcal{G}_{<}, B=\exp \mathcal{G}_{0}$ and $M=\exp \mathcal{G}_{>}$. The physical fields lie in the zero grade subgroup $B$ and the models we seek correspond to the coset $H_{-} \backslash G / H_{+}$, for $H_{ \pm}$generated by positive/negative grade operators.

For consistency with the hamiltonian reduction formalism, the phase space of the Ginvariant WZW model is reduced by specifying the constant generators $\epsilon_{ \pm}$of grade \pm 1 . In order to derive an action for $B$, invariant under

$$
g \longrightarrow g^{\prime}=\alpha_{-} g \alpha_{+},
$$

where $\alpha_{ \pm}(z, \bar{z})$ lie in the positive/negative grade subgroup we have to introduce a set of auxiliary gauge fields $A \in \mathcal{G}_{<}$and $\bar{A} \in \mathcal{G}_{>}$transforming as

$$
A \longrightarrow A^{\prime}=\alpha_{-} A \alpha_{-}^{-1}+\alpha_{-} \partial \alpha_{-}^{-1}, \quad \bar{A} \longrightarrow \bar{A}^{\prime}=\alpha_{+}^{-1} \bar{A} \alpha_{+}+\bar{\partial} \alpha_{+}^{-1} \alpha_{+},
$$

where $\partial=\partial_{t}+\partial_{x}, \bar{\partial}=\partial_{t}-\partial_{x}$. The resulting action is the $G / H\left(=H_{-} \backslash G / H_{+}\right)$gauged WZW

$$
\begin{aligned}
S_{G / H}(g, A, \bar{A}) & =S_{W Z W}(g) \\
& -\frac{k}{4 \pi} \int d^{2} x \operatorname{Tr}\left(A\left(\bar{\partial} g g^{-1}-\epsilon_{+}\right)+\bar{A}\left(g^{-1} \partial g-\epsilon_{-}\right)+A g \bar{A} g^{-1}\right) .
\end{aligned}
$$

Since the action $S_{G / H}$ is $H$-invariant, we may choose $\alpha_{-}=N^{-1}$ and $\alpha_{+}=M^{-1}$. From the orthogonality of the graded subpaces, i.e. $\operatorname{Tr}\left(\mathcal{G}_{i} \mathcal{G}_{j}\right)=0, i+j \neq 0$, we find

$$
\begin{aligned}
S_{G / H}(g, A, \bar{A}) & =S_{G / H}\left(B, A^{\prime}, \bar{A}^{\prime}\right) \\
& =S_{W Z W}(B)-\frac{k}{4 \pi} \int d^{2} x \operatorname{Tr}\left[-A^{\prime} \epsilon_{+}-\bar{A}^{\prime} \epsilon_{-}+A^{\prime} B \bar{A}^{\prime} B^{-1}\right],
\end{aligned}
$$

where we have introduced the WZW model action

$$
S_{W Z W}=-\frac{k}{4 \pi} \int d^{2} x \operatorname{Tr}\left(g^{-1} \partial g g^{-1} \bar{\partial} g\right)+\frac{k}{24 \pi} \int_{D} \epsilon^{i j k} \operatorname{Tr}\left(g^{-1} \partial_{i} g g^{-1} \partial_{j} g g^{-1} \partial_{k} g\right) d^{3} x .
$$

Performing the integration over the auxiliary fields $A$ and $\bar{A}$, we find the effective action

$$
S=S_{W Z W}(B)-\frac{k}{2 \pi} \int \operatorname{Tr}\left(\epsilon_{+} B \epsilon_{-} B^{-1}\right) d^{2} x
$$

which describes integrable perturbations of the $\mathcal{G}_{0}$-WZNW model. Those perturbations are classified in terms of the possible constant grade \pm 1 operators $\epsilon_{ \pm}$. The equations of motion associated to action (2.6) are

$$
\bar{\partial}\left(B^{-1} \partial B\right)+\left[\epsilon_{-}, B^{-1} \epsilon_{+} B\right]=0, \quad \partial\left(\bar{\partial} B B^{-1}\right)-\left[\epsilon_{+}, B \epsilon_{-} B^{-1}\right]=0
$$

For the $\mathcal{G}=\hat{S L}(3)$ case with homogeneous gradation, $Q=d$ and $\epsilon_{ \pm}=\mu \lambda_{2} \cdot H^{( \pm 1)}$ and $B=$ nam, where

$$
n=e^{\tilde{\tilde{1}}_{1} E_{-\alpha_{1}}} e^{\tilde{\tilde{2}}_{2} E_{-\alpha_{2}}} e^{\tilde{\chi}_{3} E_{-\alpha_{1}-\alpha_{2}}}, \quad a=e^{R_{1} \lambda_{1} \cdot H+R_{2} \lambda_{2} \cdot H}, \quad m=e^{\tilde{\psi}_{1} E_{\alpha_{1}}} e^{\tilde{\psi}_{2} E_{\alpha_{2}}} e^{\tilde{\psi}_{3} E_{\alpha_{1}+\alpha_{2}}}
$$

we find the explicit form for the action

$$
\begin{aligned}
S_{e f f} & =-\frac{k}{8 \pi} \int d z d \bar{z}\left(\frac{1}{3}\left(2 \partial R_{1} \bar{\partial} R_{1}-\partial R_{1} \bar{\partial} R_{2}-\partial R_{2} \bar{\partial} R_{1}+2 \partial R_{2} \bar{\partial} R_{2}\right)\right. \\
& \left.+2 \partial \tilde{\chi}_{1} \bar{\partial} \tilde{\psi}_{1} e^{R_{1}}+2 \partial \tilde{\chi}_{2} \bar{\partial} \tilde{\psi}_{2} e^{R_{2}}+2\left(\partial \tilde{\chi}_{3}-\tilde{\chi}_{2} \partial \tilde{\chi}_{1}\right)\left(\bar{\partial} \tilde{\psi}_{3}-\tilde{\psi}_{2} \bar{\partial} \tilde{\psi}_{1}\right) e^{R_{1}+R_{2}}-V\right)
\end{aligned}
$$

where $V=\mu^{2}\left(\lambda_{2}^{2}+\tilde{\psi}_{2} \tilde{\chi}_{2} e^{R_{2}}+\tilde{\psi}_{3} \tilde{\chi}_{3} e^{R_{1}+R_{2}}\right)$. 


\subsection{Reduction to the coset $G_{0} / G_{0}^{0}$}

We now introduce the subalgebra $\mathcal{G}_{0}^{0}$ such that $\left[\mathcal{G}_{0}^{0}, \epsilon_{ \pm}\right]=0$ as an additional ingredient which characterizes the symmetry of action (2.6) under chiral transformations

$$
B^{\prime}=\bar{\Omega}(\bar{z}) B \Omega(z), \quad \bar{\Omega}, \Omega \in G_{0}^{0}
$$

As consequence of symmetry under (2.10), the following chiral conservation laws are derived from the equations of motion (2.7),

$$
\bar{\partial} \operatorname{Tr}\left(X B^{-1} \partial B\right)=\partial \operatorname{Tr}\left(X \bar{\partial} B B^{-1}\right)=0, \quad X \in \mathcal{G}_{0}^{0}
$$

In order to reduce the model to the coset $G_{0} / G_{0}^{0}$, we impose the subsidiary constraints

$$
J_{X}=\operatorname{Tr}\left(X B^{-1} \partial B\right)=0, \quad \bar{J}_{X}=\operatorname{Tr}\left(X \bar{\partial} B B^{-1}\right)=0, \quad X \in \mathcal{G}_{0}^{0}
$$

which can be incorporated in the action by introducing the auxiliary gauge fields $A^{(0)}, \bar{A}^{(0)} \in$ $\mathcal{G}_{0}^{0}$. For the models where $\mathcal{G}_{0}^{0}=U(1),[10]$, [9] or $\mathcal{G}_{0}^{0}=U(1) \otimes U(1)$, [11], the action was constructed imposing symmetry under axial transformations

$$
B^{\prime \prime}=\alpha_{0}(\bar{z}, z) B \alpha_{0}(\bar{z}, z), \quad \alpha_{0} \in G_{0}^{0}
$$

and

$$
A^{\prime \prime(0)}=A^{(0)}-\alpha_{0}^{-1} \partial \alpha_{0}, \quad \bar{A}^{\prime \prime}(0)=\bar{A}^{(0)}-\alpha_{0}^{-1} \bar{\partial} \alpha_{0} .
$$

For a general non abelian $\mathcal{G}_{0}^{0}$ we can define a second grading structure $Q^{\prime}=\lambda_{1} \cdot H$ which decomposes $\mathcal{G}_{0}^{0}$ into positive, zero and negative subspaces, i.e., $\mathcal{G}_{0}^{0}=\mathcal{G}_{0}^{0,<} \oplus \mathcal{G}_{0}^{0,0} \oplus \mathcal{G}_{0}^{0,>}$. Following the same principle as in [10], [9] and [11] we seek an action invariant under

$$
B^{\prime \prime}=\gamma_{0}(\bar{z}, z) \gamma_{-}(\bar{z}, z) B \gamma_{+}(\bar{z}, z) \gamma_{0}(\bar{z}, z), \quad \gamma_{0} \in G_{0}^{0,0}, \gamma_{-} \in G_{0}^{0,<}, \gamma_{+} \in G_{0}^{0,>}
$$

and choose $\gamma_{0}(\bar{z}, z), \gamma_{-}(\bar{z}, z), \gamma_{+}(\bar{z}, z) \in G_{0}^{0}$ such that $B^{\prime \prime}=\gamma_{0} \gamma_{-} B \gamma_{+} \gamma_{0}=g_{0}^{f} \in G_{0} / G_{0}^{0}$. Note that $B$ is decomposed into the Gauss form according to the second grading structure $Q^{\prime}$. Denote $\Gamma_{-}=\gamma_{0} \gamma_{-}$and $\Gamma_{+}=\gamma_{+} \gamma_{0}$. Then the action

$$
\begin{aligned}
S\left(B, A^{(0)}, \bar{A}^{(0)}\right) & =S\left(g_{0}^{f}, A^{(0)}, \bar{A}^{(0)}\right)=S_{W Z W}(B)-\frac{k}{2 \pi} \int \operatorname{Tr}\left(\epsilon_{+} B \epsilon_{-} B^{-1}\right) d^{2} x \\
& -\frac{k}{2 \pi} \int \operatorname{Tr}\left(A^{(0)} \bar{\partial} B B^{-1}+\bar{A}^{(0)} B^{-1} \partial B+A^{(0)} B \bar{A}^{(0)} B^{-1}+A_{0}^{(0)} \bar{A}_{0}^{(0)}\right) d^{2} x
\end{aligned}
$$

is invariant under the transformations $B^{\prime}=\Gamma_{-} B \Gamma_{+}$,

$$
\begin{aligned}
& A^{\prime 0}{ }_{0}=A_{0}^{(0)}-\gamma_{0}^{-1} \partial \gamma_{0}, \quad \bar{A}_{0}^{\prime 0}=\bar{A}_{0}^{(0)}-\gamma_{0}^{-1} \bar{\partial} \gamma_{0}, \\
& A^{\prime(0)}=\Gamma_{-} A_{(0)} \Gamma_{-}^{-1}-\partial \Gamma_{-} \Gamma_{-}^{-1}, \quad \bar{A}^{\prime(0)}=\Gamma_{+}^{-1} \bar{A}_{(0)} \Gamma_{+}-\Gamma_{+}^{-1} \bar{\partial} \Gamma_{+},
\end{aligned}
$$

where $A^{(0)}=A_{0}^{(0)}+A_{-}^{(0)}$ and $\bar{A}^{(0)}=\bar{A}_{0}^{(0)}+\bar{A}_{+}^{(0)}$ and $A_{0}^{(0)}, \bar{A}_{0}^{(0)} \in \mathcal{G}_{0}^{0,0}, A_{-}^{(0)} \in \mathcal{G}_{0}^{0,<}, \bar{A}_{+}^{(0)} \in \mathcal{G}_{0}^{0,>}$. 
Let us apply the above gauge fixing procedure for the simplest case of $\mathcal{G}_{0}=S L(3, R), \quad Q=$ $d, \quad \epsilon_{ \pm}=\mu \lambda_{2} \cdot H^{( \pm 1)}$ and $\mathcal{G}_{0}^{0}=S L(2, R) \otimes U(1)$, i.e., for IM defined on the coset $\Gamma_{-} \backslash S L(3) / \Gamma_{+}$ where $\Gamma_{ \pm}=\exp \left(\tilde{\chi}_{ \pm} E_{ \pm \alpha_{1}}\right) \exp \left(\frac{1}{2} \lambda_{i} \cdot H R_{i}\right)$. Hence the auxiliary fields $A_{0}^{(0)}, \bar{A}_{0}^{(0)}, A_{-}^{(0)}$ and $\bar{A}_{+}^{(0)}$ can be parametrized as follows

$$
\begin{aligned}
& A_{0}^{(0)}=a_{1} \lambda_{1} \cdot H+a_{2}\left(\lambda_{2}-\lambda_{1}\right) \cdot H, \quad \bar{A}_{0}^{(0)}=\bar{a}_{1} \lambda_{1} \cdot H+\bar{a}_{2}\left(\lambda_{2}-\lambda_{1}\right) \cdot H, \\
& A_{-}^{(0)}=a_{21} E_{-\alpha_{1}}^{(0)}, \quad \bar{A}_{+}^{(0)}=\bar{a}_{12} E_{\alpha_{1}}^{(0)},
\end{aligned}
$$

where $a_{i}(z, \bar{z}), \bar{a}_{i}(z, \bar{z}), a_{21}(z, \bar{z})$ and $\bar{a}_{12}(z, \bar{z})$ are arbitrary functions and

$$
g_{0}^{f}=e^{\chi_{1} E_{-\alpha_{1}}+\chi_{2} E_{-\alpha_{1}-\alpha_{2}}} e^{\psi_{1} E_{\alpha_{1}}+\psi_{2} E_{\alpha_{1}+\alpha_{2}}} .
$$

The relation between the fields $\tilde{\psi}_{i}, \tilde{\chi}_{i}, R_{i}$ parametrizing the group element (2.8) and the physical fields of the gauged model $\psi_{1}, \chi_{1}, \psi_{2}, \chi_{2}$ parametrizing $g_{0}^{f}$ is given by

$$
B=e^{\frac{1}{2} R_{1} \lambda_{1} \cdot H+\frac{1}{2} R_{2} \lambda_{2} \cdot H} e^{\chi_{3} E_{-\alpha_{1}}}\left(g_{0}^{f}\right) e^{\psi_{3} E_{\alpha_{1}}} e^{\frac{1}{2} R_{1} \lambda_{1} \cdot H+\frac{1}{2} R_{2} \lambda_{2} \cdot H}
$$

or in components,

$$
\begin{array}{cl}
\tilde{\chi}_{1}=\chi_{3} e^{-\frac{1}{2} R_{1}}, & \tilde{\psi}_{1}=\psi_{3} e^{-\frac{1}{2} R_{1}}, \\
\tilde{\chi}_{2}=\chi_{2} e^{-\frac{1}{2} R_{2}}, & \tilde{\psi}_{2}=\psi_{2} e^{-\frac{1}{2} R_{2}}, \\
\tilde{\chi}_{3}=\chi_{1} e^{-\frac{1}{2}\left(R_{1}+R_{2}\right)}, & \tilde{\psi}_{3}=\psi_{1} e^{-\frac{1}{2}\left(R_{1}+R_{2}\right)} .
\end{array}
$$

In order to calculate the path integral over the auxiliary gauge fields (2.16) we first simplify the last term in eqn. (2.14)

$$
\begin{aligned}
& \operatorname{Tr}\left(A_{0}^{(0)} \bar{A}_{0}^{(0)}+A^{(0)} g_{0}^{f} \bar{A}^{(0)} g_{0}^{f-1}+A^{(0)} \bar{\partial} g_{0}^{f} g_{0}^{f-1}+\bar{A}^{(0)} g_{0}^{f-1} \partial g_{0}^{f}\right) \\
& =\bar{a}_{i} M_{i j} a_{j}+\bar{a}_{i} N_{i}+\bar{N}_{i} a_{i}+\bar{a}_{12} a_{21}\left(1+\psi_{2} \chi_{2}\right)-\bar{a}_{12} \psi_{2} \partial \chi_{1}-a_{21} \chi_{2} \bar{\partial} \psi_{1}
\end{aligned}
$$

where we have introduced the matrix $M$,

$$
M=\left(\begin{array}{cc}
\frac{4}{3}+\psi_{1} \chi_{1} & -\frac{2}{3} \\
-\frac{2}{3} & \frac{4}{3}+\psi_{2} \chi_{2}
\end{array}\right)
$$

and the vectors $N$ and $\bar{N}$,

$$
\bar{N}=\left(-\left(\bar{\partial} \psi_{1}-\bar{a}_{12} \psi_{2}\right) \chi_{1}, \quad-\left(\chi_{2} \bar{\partial} \psi_{2}\right)\right), \quad N=\left(\begin{array}{c}
-\left(\partial \chi_{1}-a_{21} \chi_{2}\right) \psi_{1} \\
-\left(\psi_{2} \partial \chi_{2}\right)
\end{array}\right)
$$

We first evaluate the integral over $a_{i}$ and $\bar{a}_{i}$ in the partition function

$$
Z=\int \mathcal{D} B \mathcal{D} A_{0}^{(0)} \mathcal{D} \bar{A}_{0}^{(0)} \mathcal{D} A_{-}^{(0)} \mathcal{D} \bar{A}_{+}^{(0)} e^{-S\left(B, A^{(0)}, \bar{A}^{(0)}\right)}
$$

i.e., the Gaussian integral

$$
\int D \bar{a} D a e^{\int(\bar{a} M a+\bar{a} N+\bar{N} a)}=\text { const. } \cdot e^{-\left(\bar{N} M^{-1} N\right)}
$$


Taking into account the explicit form of $\bar{N} M^{-1} N$,i.e.,

$$
\begin{aligned}
N M^{-1} N & =\frac{4 \Delta}{3 D} a_{21} \bar{a}_{12}+2 \frac{\chi_{2} a_{21}}{3 D}\left(\chi_{2} \psi_{1} \bar{\partial} \psi_{2}-2\left(1+\psi_{2} \chi_{2}\right) \bar{\partial} \psi_{1}\right) \\
& +2 \frac{\psi_{2} \bar{a}_{12}}{3 D}\left(\psi_{2} \chi_{1} \partial \chi_{2}-2\left(1+\psi_{2} \chi_{2}\right) \partial \chi_{1}\right) \\
& +\frac{1}{3 D}\left(-\left(4+3 \psi_{2} \chi_{2}\right) \psi_{1} \chi_{1} \bar{\partial} \psi_{1} \partial \chi_{1}-2 \chi_{1} \psi_{2} \partial \chi_{2} \bar{\partial} \psi_{3}\right. \\
& \left.-2 \chi_{2} \psi_{1} \bar{\partial} \psi_{2} \partial \chi_{1}-\left(4+3 \psi_{1} \chi_{1}\right) \psi_{2} \chi_{2} \bar{\partial} \psi_{2} \partial \chi_{2}\right) \\
\Delta & =\left(1+\psi_{2} \chi_{2}\right)^{2}+\psi_{1} \chi_{1}\left(1+\frac{3}{4} \psi_{2} \chi_{2}\right) \\
D & =\operatorname{DetM}=\frac{4}{3}\left(1+\psi_{1} \chi_{1}+\psi_{2} \chi_{2}+\frac{3}{4} \psi_{1} \chi_{1} \psi_{2} \chi_{2}\right)
\end{aligned}
$$

and eqn. (2.20), we integrate over $a_{21}$ and $\bar{a}_{12}$. As a result we derive the effective action for the gauge fixed model ${ }^{1}$ :

$$
\begin{gathered}
S_{e f f}=-\frac{k}{2 \pi} \int d z d \bar{z}\left(\frac { 1 } { \Delta } \left(\bar{\partial} \psi_{2} \partial \chi_{2}\left(1+\psi_{1} \chi_{1}+\psi_{2} \chi_{2}\right)+\bar{\partial} \psi_{1} \partial \chi_{1}\left(1+\psi_{2} \chi_{2}\right)\right.\right. \\
\left.\left.-\frac{1}{2}\left(\psi_{2} \chi_{1} \bar{\partial} \psi_{1} \partial \chi_{2}+\chi_{2} \psi_{1} \bar{\partial} \psi_{2} \partial \chi_{1}\right)\right)-V\right)
\end{gathered}
$$

where $V=\mu^{2}\left(\frac{2}{3}+\psi_{1} \chi_{1}+\psi_{2} \chi_{2}\right)$. It appears to be the simplest generalization of the complex sine-Gordon model [23] and belongs to the same hierarchy of the Fordy-Kulish (multicomponent ) non-linear Schroedinger model [20], [22]. One can also derive it as a further hamiltonian reduction of the $A_{2}^{(1)}$-Homogeneous sine-Gordon model [21].

It is worthwhile to mention that the classical integrability of the gauged fixed model (2.25) is a consequence of the integrability of the corresponding ungauged model (2.9). The zero curvature (Lax) representation of the IM (2.9)(or equivalently (2.7)) has the well known form:

$$
\partial \overline{\mathcal{A}}-\bar{\partial} \mathcal{A}-[\mathcal{A}, \overline{\mathcal{A}}]=0, \quad \mathcal{A}, \overline{\mathcal{A}} \in \oplus_{i=0, \pm 1} \mathcal{G}_{i}
$$

with

$$
\mathcal{A}=-B \epsilon_{-} B^{-1}, \quad \overline{\mathcal{A}}=\epsilon_{+}+\bar{\partial} B B^{-1}
$$

We next impose the constraints (2.12) on the group element $B$ (2.8), i.e. substituting the non-physical fields $R_{1}, R_{2}, \tilde{\psi}_{1}$ and $\tilde{\chi}_{1}$ by their nonlocal expressions obtained as a solution of the constraints (2.12) (see eqns. (3.34) and (3.35) for their explicit form). This gives the Lax connection $\mathcal{A}, \overline{\mathcal{A}}$ for the gauged model (2.25). It can be easily verified that substituting (2.27) into (2.26) and taking into account (2.12) (or (3.34) and (3.35)), one reproduces the equations of motion derived from the action (2.25). Then the existence of an infinite set (of

\footnotetext{
${ }^{1}$ since in this paper we are interested in the solution and symmetries of the classical gauge fixed model we are consistently neglecting all the quantum contributions to $S_{\text {eff }}$ comming from the determinant factors, ghost field action, counter terms, etc
} 
commuting) conserved charges $P_{m}, \quad m=0,1, \cdots$ is a simple consequence of eqn. (2.26), namely,

$$
P_{m}(t)=\operatorname{Tr}(T(t))^{m}, \quad \partial_{t} P_{m}=0, \quad T(t)=\lim _{L \rightarrow \infty} \mathcal{P} \exp \int_{-L}^{L} \mathcal{A}_{x}(t, x) d x
$$

Hence the above described procedure for derivation of the NA affine Toda model (2.25) as gauged $G / H$ two loop WZW models leads to integrable model by construction.

\section{$3 \quad$ Symmetries}

\subsection{Chiral symmetries in the Group $G_{0}$}

Let us now discuss the symmetry structure of the ungauged IM on the $S L(3)$ group given by eqn. (2.9). It is generated by the chiral transformation (2.10), i.e., $B^{\prime}=\bar{\Omega}(\bar{z}) B \Omega(z), \quad \bar{\Omega}, \Omega \in$ $G_{0}^{0}=S L(2) \otimes U(1)$ generated by $\left\{\lambda_{1} \cdot H, \lambda_{2} \cdot H, E_{ \pm \alpha_{1}}\right\}$. We make use of the defining representation of $S L(3)$ in terms of $3 \times 3$ matrices to parametrize the zero grade group element $B(2.8)$ in terms of the Gauss decomposition, i.e.,

$$
\begin{aligned}
& B_{1,1}=e^{\frac{2}{3} R_{1}+\frac{1}{3} R_{2}}, \quad B_{1,2}=B_{1,1} \tilde{\psi}_{1}, \quad B_{1,3}=B_{1,1} \tilde{\psi}_{3}, \quad B_{2,3}=B_{1,1}\left(e^{-R_{1}} \tilde{\psi}_{2}+\tilde{\chi}_{1} \tilde{\psi}_{3}\right), \\
& B_{2,1}=B_{1,1} \tilde{\chi}_{1}, \quad B_{2,2}=B_{1,1}\left(e^{-R_{1}}+\tilde{\psi}_{1} \tilde{\chi}_{1}\right), \quad B_{3,1}=B_{1,1} \tilde{\chi}_{3}, \\
& B_{3,2}=B_{1,1}\left(e^{-R_{1}} \tilde{\chi}_{2}+\tilde{\psi}_{1} \tilde{\chi}_{3}\right), \quad B_{3,3}=B_{1,1}\left(\tilde{\psi}_{1} \tilde{\chi}_{1}+e^{-R_{1}} \tilde{\psi}_{2} \tilde{\chi}_{2}+e^{-R_{1}-R_{2}}\right),
\end{aligned}
$$

We also find for the chiral symmetry transformations

$$
\begin{aligned}
& \Omega_{1,1}=e^{\frac{2}{3} \epsilon_{1}+\frac{1}{3} \epsilon_{2}}, \quad \Omega_{1,2}=\Omega_{1,1} \epsilon_{+}, \quad \Omega_{2,1}=\Omega_{1,1} \epsilon_{-}, \\
& \Omega_{2,2}=\Omega_{1,1}\left(e^{-\epsilon_{1}}+\epsilon_{-} \epsilon_{+}\right), \quad \Omega_{3,3}=\Omega_{1,1} e^{-\epsilon_{1}-\epsilon_{2}} \\
& \Omega_{1,3}=\Omega_{2,3}=\Omega_{3,2}=\Omega_{3,1}=0
\end{aligned}
$$

and $\bar{\Omega}=\Omega(\epsilon \rightarrow \bar{\epsilon})$ where $\epsilon=\epsilon(z)$ and $\bar{\epsilon}=\bar{\epsilon}(\bar{z})$. The infinitesimal chiral transformations for (2.10) yields the following field transformations,

$$
\begin{aligned}
\delta R_{1} & =\epsilon_{1}+\bar{\epsilon}_{1}+2 \epsilon_{-} \tilde{\psi}_{1}+2 \bar{\epsilon}_{+} \tilde{\chi}_{1}, \\
\delta R_{2} & =\epsilon_{2}+\bar{\epsilon}_{2}-\epsilon_{-} \tilde{\psi}_{1}-\bar{\epsilon}_{+} \tilde{\chi}_{1}, \\
\delta \tilde{\psi}_{1} & =\epsilon_{+}-\epsilon_{1} \tilde{\psi}_{1}+\bar{\epsilon}_{+} e^{-R_{1}}-\epsilon_{-} \tilde{\psi}_{1}^{2}, \\
\delta \tilde{\chi}_{1} & =\bar{\epsilon}_{-}-\bar{\epsilon}_{1} \tilde{\chi}_{1}+\epsilon_{-} e^{-R_{1}}-\bar{\epsilon}_{+} \tilde{\chi}_{1}^{2}, \\
\delta \tilde{\psi}_{2} & =\epsilon_{-}\left(\tilde{\psi}_{1} \tilde{\psi}_{2}-\tilde{\psi}_{3}\right)-\epsilon_{2} \tilde{\psi}_{2}, \\
\delta \tilde{\chi}_{2} & =\bar{\epsilon}_{+}\left(\tilde{\chi}_{1} \tilde{\chi}_{2}-\tilde{\chi}_{3}\right)-\bar{\epsilon}_{2} \tilde{\chi}_{2}, \\
\delta \tilde{\psi}_{3} & =-\left(\epsilon_{1}+\epsilon_{2}\right) \tilde{\psi}_{3}+\bar{\epsilon}_{+} \tilde{\psi}_{2} e^{-R_{1}}-\epsilon_{-} \tilde{\psi}_{1} \tilde{\psi}_{3}, \\
\delta \tilde{\chi}_{3} & =-\left(\bar{\epsilon}_{1}+\bar{\epsilon}_{2}\right) \tilde{\chi}_{3}+\epsilon_{-} \tilde{\chi}_{2} e^{-R_{1}}-\bar{\epsilon}_{+} \tilde{\chi}_{1} \tilde{\chi}_{3}
\end{aligned}
$$


As a consequence of the invariance of the action (2.9) under the chiral transformations (3.30) we find Noether currents to correspond to the chiral currents (2.11) associated to the $\mathcal{G}_{0}^{0}$ subalgebra. For the explicit example of the $\mathcal{G}_{0}=S L(3)$ we have

$$
\begin{aligned}
J_{-\alpha_{1}}= & \partial \tilde{\psi}_{1}-\tilde{\psi}_{1}^{2} \partial \tilde{\chi}_{1} e^{R_{1}}+\partial \tilde{\chi}_{2}\left(\tilde{\psi}_{1} \tilde{\psi}_{2}-\tilde{\psi}_{3}\right) e^{R_{2}} \\
& +\left(\partial \tilde{\chi}_{3}-\tilde{\chi}_{2} \partial \tilde{\chi}_{1}\right)\left(\tilde{\psi}_{1} \tilde{\psi}_{2}-\tilde{\psi}_{3}\right) \tilde{\psi}_{1} e^{R_{1}+R_{2}}+\tilde{\psi}_{1} \partial R_{1}, \\
J_{\alpha_{1}}= & \partial \tilde{\chi}_{1} e^{R_{1}}-\tilde{\psi}_{2}\left(\partial \tilde{\chi}_{3}-\tilde{\chi}_{2} \partial \tilde{\chi}_{1}\right) e^{R_{1}+R_{2}}, \\
J_{\lambda_{1} \cdot H}= & \frac{1}{3}\left(2 \partial R_{1}+\partial R_{2}\right)-\tilde{\psi}_{1} \partial \tilde{\chi}_{1} e^{R_{1}}+\left(\tilde{\psi}_{1} \tilde{\psi}_{2}-\tilde{\psi}_{3}\right)\left(\partial \tilde{\chi}_{3}-\tilde{\chi}_{2} \partial \tilde{\chi}_{1}\right) e^{R_{1}+R_{2}}, \\
J_{\lambda_{2} \cdot H}= & \frac{1}{3}\left(\partial R_{1}+2 \partial R_{2}\right)-\tilde{\psi}_{2} \partial \tilde{\chi}_{2} e^{R_{2}}-\tilde{\psi}_{3}\left(\partial \tilde{\chi}_{3}-\tilde{\chi}_{2} \partial \tilde{\chi}_{1}\right) e^{R_{1}+R_{2}} \\
\bar{J}_{\alpha_{1}}= & \bar{\partial} \tilde{\chi}_{1}-\tilde{\chi}_{1}^{2} \bar{\partial} \tilde{\psi}_{1} e^{R_{1}}+\bar{\partial}_{2}\left(\tilde{\chi}_{1} \tilde{\chi}_{2}-\tilde{\chi}_{3}\right) e^{R_{2}} \\
& +\left(\bar{\partial} \tilde{\psi}_{3}-\tilde{\psi}_{2} \bar{\partial} \tilde{\psi}_{1}\right)\left(\tilde{\chi}_{1} \tilde{\chi}_{2}-\tilde{\chi}_{3}\right) \tilde{\chi}_{1} e^{R_{1}+R_{2}}+\tilde{\chi}_{1} \partial R_{1}, \\
\bar{J}_{-\alpha_{1}}= & \bar{\partial} \tilde{\psi}_{1} e^{R_{1}}-\tilde{\chi}_{2}\left(\bar{\partial} \tilde{\psi}_{3}-\tilde{\psi}_{2} \bar{\partial} \tilde{\psi}_{1}\right) e^{R_{1}+R_{2}}, \\
\bar{J}_{\lambda_{1} \cdot H}= & \frac{1}{3}\left(2 \bar{\partial} R_{1}+\bar{\partial} R_{2}\right)-\tilde{\chi}_{1} \bar{\partial} \tilde{\psi}_{1} e^{R_{1}}+\left(\tilde{\chi}_{1} \tilde{\chi}_{2}-\tilde{\chi}_{3}\right)\left(\bar{\partial} \tilde{\psi}_{3}-\tilde{\psi}_{2} \bar{\partial} \tilde{\psi}_{1}\right) e^{R_{1}+R_{2}}, \\
\bar{J}_{\lambda_{2} \cdot H}= & \frac{1}{3}\left(\bar{\partial} R_{1}+2 \bar{\partial} R_{2}\right)-\tilde{\chi}_{2} \bar{\partial} \tilde{\psi}_{2} e^{R_{2}}-\tilde{\chi}_{3}\left(\bar{\partial} \tilde{\psi}_{3}-\tilde{\psi}_{2} \bar{\partial} \tilde{\psi}_{1}\right) e^{R_{1}+R_{2}}
\end{aligned}
$$

where $\bar{\partial} J=\partial \bar{J}=0$ and $J=J_{\lambda_{1} \cdot H} h_{1}+J_{\lambda_{2} \cdot H} h_{2}+\sum_{\alpha=\alpha_{1}, \alpha_{2}, \alpha_{1}+\alpha_{2}} J_{\alpha} E_{-\alpha}+J_{-\alpha} E_{\alpha}$.

One can easily verify that the algebra of the local (chiral ) infinitesimal transformations (3.30), that leaves invariant the action of the ungauged IM $(2.9)$ is $(S L(2) \otimes U(1))_{\text {left }} \otimes$ $(S L(2) \otimes U(1))_{\text {right }}$.

\subsection{Global symmetries in the coset $G_{0} / G_{0}^{0}$}

The reduced model in the coset $G_{0} / G_{0}^{0}$ is obtained by implementing the additional constraints (2.12), i.e. by the vanishing of the chiral currents (2.12). For the $S L(3)$ example this allows the elimination of four degrees of freedom $R_{i}, \tilde{\psi}_{1}$ and $\tilde{\chi}_{1}$, i.e., taking into account (3.31) and (2.12) we find

$$
\begin{aligned}
\partial R_{1} & =2 \tilde{\psi}_{1} \partial \tilde{\chi}_{1} e^{R_{1}}-\tilde{\psi}_{2} \partial \tilde{\chi}_{2} e^{R_{2}}+\left(\partial \tilde{\chi}_{3}-\tilde{\chi}_{2} \partial \tilde{\chi}_{1}\right)\left(\tilde{\psi}_{3}-2 \tilde{\psi}_{1} \tilde{\psi}_{2}\right) e^{R_{1}+R_{2}}, \\
\partial R_{2} & =-\tilde{\psi}_{1} \partial \tilde{\chi}_{1} e^{R_{1}}+2 \tilde{\psi}_{2} \partial \tilde{\chi}_{2} e^{R_{2}}+\left(\partial \tilde{\chi}_{3}-\tilde{\chi}_{2} \partial \tilde{\chi}_{1}\right)\left(\tilde{\psi}_{3}+\tilde{\psi}_{1} \tilde{\psi}_{2}\right) e^{R_{1}+R_{2}}, \\
\partial \tilde{\psi}_{1} & =\tilde{\psi}_{3} \partial \tilde{\chi}_{2} e^{R_{2}} \\
\partial \tilde{\chi}_{1} & =\tilde{\psi}_{2}\left(\partial \tilde{\chi}_{3}-\tilde{\chi}_{2} \partial \tilde{\chi}_{1}\right) e^{R_{2}} \\
\bar{\partial} R_{1} & =2 \tilde{\chi}_{1} \bar{\partial} \tilde{\psi}_{1} e^{R_{1}}-\tilde{\chi}_{2} \bar{\partial} \tilde{\psi}_{2} e^{R_{2}}+\left(\bar{\partial} \tilde{\psi}_{3}-\tilde{\psi}_{2} \bar{\partial} \tilde{\psi}_{1}\right)\left(\tilde{\chi}_{3}-2 \tilde{\chi}_{1} \tilde{\chi}_{2}\right) e^{R_{1}+R_{2}}, \\
\bar{\partial} R_{2} & =-\tilde{\chi}_{1} \bar{\partial} \tilde{\psi}_{1} e^{R_{1}}+2 \tilde{\chi}_{2} \bar{\partial} \tilde{\psi}_{2} e^{R_{2}}+\left(\bar{\partial} \tilde{\psi}_{3}-\tilde{\psi}_{2} \bar{\partial} \tilde{\psi}_{1}\right)\left(\tilde{\chi}_{3}+\tilde{\chi}_{1} \tilde{\chi}_{2}\right) e^{R_{1}+R_{2}}, \\
\bar{\partial} \tilde{\psi}_{1} & =\tilde{\chi}_{2}\left(\bar{\partial} \tilde{\psi}_{3}-\tilde{\psi}_{2} \bar{\partial} \tilde{\psi}_{1}\right) e^{R_{2}}, \\
\bar{\partial} \tilde{\chi}_{1} & =\tilde{\chi}_{3} \bar{\partial} \tilde{\psi}_{2} e^{R_{2}}
\end{aligned}
$$

In order to eliminate the unphysical fields $R_{i}, \tilde{\psi}_{1}$ and $\tilde{\chi}_{1}$ we recall eqn. (2.19) relating the fields of the gauged and ungauged models (2.25) and (2.9) respectively. In terms of these 
variables the transformations (3.30) become,

$$
\begin{aligned}
\delta \psi_{1} & =\frac{1}{2}\left(-\epsilon_{1}-\epsilon_{2}+\bar{\epsilon}_{1}+\bar{\epsilon}_{2}\right) \psi_{1}-\frac{1}{2} \epsilon_{-} \psi_{1} \tilde{\psi}_{1}+\bar{\epsilon}_{+}\left(\psi_{2} e^{-\frac{1}{2} R_{1}}+\frac{1}{2} \psi_{1} \tilde{\chi}_{1}\right), \\
\delta \chi_{1} & =\frac{1}{2}\left(\epsilon_{1}+\epsilon_{2}-\bar{\epsilon}_{1}-\bar{\epsilon}_{2}\right) \chi_{1}-\frac{1}{2} \bar{\epsilon}_{+} \chi_{1} \tilde{\chi}_{1}+\epsilon_{-}\left(\chi_{2} e^{-\frac{1}{2} R_{1}}+\frac{1}{2} \chi_{1} \tilde{\psi}_{1}\right), \\
\delta \psi_{2} & =\epsilon_{-}\left(\frac{1}{2} \psi_{2} \tilde{\psi}_{1}-\psi_{1} e^{-\frac{1}{2} R_{1}}\right)-\frac{1}{2} \bar{\epsilon}_{+} \psi_{2} \tilde{\chi}_{1}+\frac{1}{2}\left(-\epsilon_{2}+\bar{\epsilon}_{2}\right) \psi_{2}, \\
\delta \chi_{2} & =\bar{\epsilon}_{+}\left(\frac{1}{2} \chi_{2} \tilde{\chi}_{1}-\chi_{1} e^{-\frac{1}{2} R_{1}}\right)-\frac{1}{2} \epsilon_{-} \chi_{2} \tilde{\psi}_{1}+\frac{1}{2}\left(\epsilon_{2}-\bar{\epsilon}_{2}\right) \chi_{2},
\end{aligned}
$$

where $\epsilon_{i}, \bar{\epsilon}_{i}, \epsilon_{ \pm}$and $\bar{\epsilon}_{ \pm}$satisfy now certain restrictions ${ }^{2}$ (see eqn. (3.47) below ) which forces them to be constants. By simplifying eqn. (3.32) we obtain the nonlocal fields $R_{i}$ in the form:

$$
\begin{aligned}
\partial R_{1} & =\frac{\psi_{1} \partial \chi_{1}}{\Delta}\left(1+\frac{3}{2} \psi_{2} \chi_{2}\right)-\frac{\psi_{2} \partial \chi_{2}}{\Delta}\left(\Delta_{2}+\frac{3}{2} \psi_{1} \chi_{1}\right), \\
\partial R_{2} & =\frac{\psi_{1} \partial \chi_{1}}{\Delta}+\frac{\psi_{2} \partial \chi_{2}}{\Delta}\left(2 \Delta_{2}+\frac{3}{2} \psi_{1} \chi_{1}\right), \\
\bar{\partial} R_{1} & =\frac{\chi_{1} \bar{\partial} \psi_{1}}{\Delta}\left(1+\frac{3}{2} \psi_{2} \chi_{2}\right)-\frac{\chi_{2} \bar{\partial} \psi_{2}}{\Delta}\left(\Delta_{2}+\frac{3}{2} \psi_{1} \chi_{1}\right), \\
\bar{\partial} R_{2} & =\frac{\chi_{1} \bar{\partial} \psi_{1}}{\Delta}+\frac{\chi_{2} \bar{\partial} \psi_{2}}{\Delta}\left(2 \Delta_{2}+\frac{3}{2} \psi_{1} \chi_{1}\right)
\end{aligned}
$$

where $\Delta=\left(1+\psi_{2} \chi_{2}\right)^{2}+\psi_{1} \chi_{1}\left(1+\frac{3}{4} \psi_{2} \chi_{2}\right), \quad \Delta_{2}=1+\psi_{2} \chi_{2}$. In addition we find

$$
\begin{aligned}
\partial \tilde{\chi}_{1} & =\frac{\psi_{2}}{\Delta}\left(\partial \chi_{1} \Delta_{2}-\frac{1}{2} \chi_{1} \psi_{2} \partial \chi_{2}\right) e^{-\frac{1}{2} R_{1}} \\
\partial \tilde{\psi}_{1} & =\frac{\psi_{1}}{\Delta}\left(\partial \chi_{2}\left(1+\psi_{1} \chi_{1}+\psi_{2} \chi_{2}\right)-\frac{1}{2} \chi_{2} \psi_{1} \partial \chi_{1}\right) e^{-\frac{1}{2} R_{1}} \\
\bar{\partial} \tilde{\psi}_{1} & =\frac{\chi_{2}}{\Delta}\left(\bar{\partial} \psi_{1} \Delta_{2}-\frac{1}{2} \psi_{1} \chi_{2} \bar{\partial} \psi_{2}\right) e^{-\frac{1}{2} R_{1}} \\
\bar{\partial} \tilde{\chi}_{1} & =\frac{\chi_{1}}{\Delta}\left(\bar{\partial} \psi_{2}\left(1+\psi_{1} \chi_{1}+\psi_{2} \chi_{2}\right)-\frac{1}{2} \chi_{1} \psi_{2} \bar{\partial} \psi_{1}\right) e^{-\frac{1}{2} R_{1}}
\end{aligned}
$$

We next define the conserved topological currents

$$
j_{R_{i}, \mu}=\epsilon_{\mu \nu} \partial_{\nu} R_{i}, \quad i=1,2, \quad j_{\tilde{\psi}_{1}, \mu}=\epsilon_{\mu \nu} \partial_{\nu} \tilde{\psi}_{1}, \quad j_{\tilde{\chi}_{1}, \mu}=\epsilon_{\mu \nu} \partial_{\nu} \tilde{\chi}_{1},
$$

Using the equations of motion derived from (2.25), one can confirm the following conservation laws

$$
\bar{\partial} j=\partial \bar{j}, \quad j=j_{\tilde{\psi}_{1}}, \quad j_{\tilde{\chi}_{1}}, \quad j=j_{R_{i}}, i=1,2,
$$

where $j=\frac{1}{2}\left(j_{0}+j_{1}\right), \quad \bar{j}=\frac{1}{2}\left(j_{0}-j_{1}\right)$. Notice that (3.34) and (3.35) define the non local fields $R_{1}, R_{2}, \tilde{\psi}_{1}, \tilde{\chi}_{1}$ in terms of the physical fields $\psi_{1}, \psi_{2}, \chi_{1}$ and $\chi_{2}$. Hence the conservation of the currents defined by the r.h.s. of (3.34) and (3.35) is non-trivial and requires the use of the equations of motion.

\footnotetext{
${ }^{2}$ comming from the requirement of the invariance of constraints equations (3.32)
} 


\subsection{Algebra of the global symmetries}

The simplest way to derive the algebra of symmetries of gauged IM (2.25) (generated by transformations (3.33) ) is to realize the charges of non-chiral conserved currents (3.34), (3.35) and (3.36):

$$
\begin{aligned}
Q_{1} & =\frac{1}{3} \int\left(2 \partial_{x} R_{1}+\partial_{x} R_{2}\right) d x, \quad Q_{2}=\frac{1}{3} \int\left(\partial_{x} R_{1}+2 \partial_{x} R_{2}\right) d x \\
Q_{\tilde{\chi}_{1}} & =Q_{-}=\int \partial_{x} \tilde{\chi}_{1} d x, \quad Q_{\tilde{\psi}_{1}}=Q_{+}=\int \partial_{x} \tilde{\psi}_{1} d x
\end{aligned}
$$

in terms of the canonical momenta

$$
\begin{aligned}
\Pi_{\psi_{1}} & =\frac{\delta \mathcal{L}}{\delta \dot{\psi}_{1}}=\frac{-k}{2 \pi}\left(\frac{\partial \chi_{1}}{\Delta}\left(1+\psi_{2} \chi_{2}\right)-\frac{1}{2} \frac{\partial \chi_{2}}{\Delta} \chi_{1} \psi_{2}\right) \\
\Pi_{\psi_{2}} & =\frac{\delta \mathcal{L}}{\delta \dot{\psi}_{2}}=\frac{-k}{2 \pi}\left(\frac{\partial \chi_{2}}{\Delta}\left(1+\psi_{1} \chi_{1}+\psi_{2} \chi_{2}\right)-\frac{1}{2} \frac{\partial \chi_{1}}{\Delta} \chi_{2} \psi_{1}\right), \\
\Pi_{\chi_{1}} & =\frac{\delta \mathcal{L}}{\delta \dot{\chi}_{1}}=\frac{-k}{2 \pi}\left(\frac{\bar{\partial} \psi_{1}}{\Delta}\left(1+\psi_{2} \chi_{2}\right)-\frac{1}{2} \frac{\bar{\partial} \psi_{2}}{\Delta} \chi_{2} \psi_{1}\right) \\
\Pi_{\chi_{2}} & =\frac{\delta \mathcal{L}}{\delta \dot{\chi}_{2}}=\frac{-k}{2 \pi}\left(\frac{\bar{\partial} \psi_{2}}{\Delta}\left(1+\psi_{1} \chi_{1}+\psi_{2} \chi_{2}\right)-\frac{1}{2} \frac{\bar{\partial} \psi_{1}}{\Delta} \chi_{1} \psi_{2}\right),
\end{aligned}
$$

By substituting (3.39) in eqns. (3.34) and (3.35) we obtain

$$
\begin{aligned}
\partial R_{1} & =\frac{-2 \pi}{k}\left(\psi_{1} \Pi_{\psi_{1}}-\psi_{2} \Pi_{\psi_{2}}\right), \quad \bar{\partial} R_{1}=\frac{-2 \pi}{k}\left(\chi_{1} \Pi_{\chi_{1}}-\chi_{2} \Pi_{\chi_{2}}\right), \\
\partial R_{2} & =\frac{-2 \pi}{k}\left(\psi_{1} \Pi_{\psi_{1}}+2 \psi_{2} \Pi_{\psi_{2}}\right), \quad \bar{\partial} R_{2}=\frac{-2 \pi}{k}\left(\chi_{1} \Pi_{\chi_{1}}+2 \chi_{2} \Pi_{\chi_{2}}\right), \\
\partial \tilde{\chi}_{1} & =\frac{-2 \pi}{k} \psi_{2} \Pi_{\psi_{1}} e^{-\frac{1}{2} R_{1}}, \quad \bar{\partial} \tilde{\chi}_{1}=\frac{-2 \pi}{k} \chi_{1} \Pi_{\chi_{2}} e^{-\frac{1}{2} R_{1}} \\
\partial \tilde{\psi}_{1} & =\frac{-2 \pi}{k} \psi_{1} \Pi_{\psi_{2}} e^{-\frac{1}{2} R_{1}}, \quad \bar{\partial} \tilde{\psi}_{1}=\frac{-2 \pi}{k} \chi_{2} \Pi_{\chi_{1}} e^{-\frac{1}{2} R_{1}}
\end{aligned}
$$

In order to calculate the field transformations

$$
\begin{aligned}
\delta_{ \pm} \psi_{i} & =\left\{Q_{ \pm}, \psi_{i}\right\} \epsilon_{ \pm}^{g}, \quad \delta_{ \pm} \chi_{i}=\left\{Q_{ \pm}, \chi_{i}\right\} \epsilon_{ \pm}^{g}, \\
\delta_{j} \psi_{i} & =\left\{Q_{j}, \psi_{i}\right\} \epsilon_{j}^{g}, \quad \delta_{j} \chi_{i}=\left\{Q_{j}, \chi_{i}\right\} \epsilon_{j}^{g}
\end{aligned}
$$

we use the canonical Poisson brackets (PB) (where $\epsilon^{g}$ are constant parameters)

$$
\left\{\Pi_{\phi_{i}}(x), \phi_{k}(y)\right\}=\delta_{i k} \delta(x-y), \quad \phi_{k}=\psi_{i}, \chi_{i}
$$

and also few consequences of (3.42) and (3.40):

$$
\begin{aligned}
& \left\{\partial_{x} R_{1}(x), \psi_{i}(y)\right\}=(-1)^{i+1} \psi_{i}(y) \delta(x-y), \quad\left\{\partial_{x} R_{1}(x), \chi_{i}(y)\right\}=(-1)^{i} \chi_{i}(y) \delta(x-y) \\
& \left\{\partial_{x} R_{2}(x), \psi_{2}(y)\right\}=2 \psi_{2}(y) \delta(x-y), \quad\left\{\partial_{x} R_{2}(x), \chi_{2}(y)\right\}=-2 \chi_{2}(y) \delta(x-y)
\end{aligned}
$$


etc. Evaluating the corresponding PBs we find the field transformations we seek:

$$
\begin{aligned}
& \delta_{+} \chi_{1}=\frac{1}{2}\left(\chi_{2} e^{-\frac{1}{2} R_{1}}+\frac{1}{2} \chi_{1} \tilde{\psi}_{1}\right) \epsilon_{+}^{g}, \quad \delta_{-} \chi_{1}=\frac{1}{4} \chi_{1} \tilde{\chi}_{1} \epsilon_{-}^{g}, \\
& \delta_{+} \psi_{1}=-\frac{1}{4} \psi_{1} \tilde{\psi}_{1} \epsilon_{+}^{g}, \quad \delta_{-} \psi_{1}=-\frac{1}{2}\left(\psi_{2} e^{-\frac{1}{2} R_{1}}+\frac{1}{2} \psi_{1} \tilde{\chi}_{1}\right) \epsilon_{-}^{g}, \\
& \delta_{+} \chi_{2}=-\frac{1}{4} \chi_{2} \tilde{\psi}_{1} \epsilon_{+}^{g}, \quad \delta_{-} \chi_{2}=\frac{1}{2}\left(\chi_{1} e^{-\frac{1}{2} R_{1}}-\frac{1}{2} \chi_{2} \tilde{\chi}_{1}\right) \epsilon_{-}^{g}, \\
& \delta_{+} \psi_{2}=-\frac{1}{2}\left(\psi_{1} e^{-\frac{1}{2} R_{1}}-\frac{1}{2} \psi_{2} \tilde{\psi}_{1}\right) \epsilon_{+}^{g}, \quad \delta_{-} \psi_{2}=\frac{1}{4} \psi_{2} \tilde{\chi}_{1} \epsilon_{-}^{g}
\end{aligned}
$$

Note that the above transformations are nonlocal due to the presence of $\tilde{\psi}_{1}, \tilde{\chi}_{1}$ and $R_{1}$ which are defined in terms of integrals of the fields $\psi_{i}, \chi_{i}$ and their derivatives:

$$
\begin{array}{r}
\tilde{\psi}_{1}(x)=\frac{1}{2} \int \epsilon(x-y)\left(\psi_{1}(y) \Pi_{\psi_{2}}(y)-\chi_{2}(y) \Pi_{\chi_{1}}(y)\right) e^{-\frac{1}{2} R_{1}(y)} d y \\
R_{1}(x)=\frac{1}{2} \int \epsilon(x-y)\left(\psi_{1}(y) \Pi_{\psi_{1}}(y)-\psi_{2}(y) \Pi_{\psi_{2}}(y)-\chi_{1}(y) \Pi_{\chi_{1}}(y)+\chi_{2}(y) \Pi_{\chi_{2}}(y)\right) d y
\end{array}
$$

and $\tilde{\chi}_{1}=\tilde{\psi}_{1}\left(\psi_{1} \leftrightarrow \psi_{2}, \quad \chi_{1} \leftrightarrow \chi_{2}\right)$. Instead the transformations generated by the charges $Q_{1}$ and $Q_{2}$ have the following simple, local and linear in the fields form:

$$
\begin{aligned}
& \delta_{1} \chi_{1}=-\chi_{1} \epsilon_{1}^{g}, \quad \delta_{2} \chi_{1}=-\chi_{1} \epsilon_{2}^{g}, \\
& \delta_{1} \psi_{1}=\psi_{1} \epsilon_{1}^{g}, \quad \delta_{2} \psi_{1}=\psi_{1} \epsilon_{2}^{g}, \\
& \delta_{1} \chi_{2}=0, \quad \delta_{2} \chi_{2}=-\chi_{2} \epsilon_{2}^{g}, \\
& \delta_{1} \psi_{2}=0, \quad \delta_{2} \psi_{2}=\psi_{2} \epsilon_{2}^{g}
\end{aligned}
$$

Observe that the above transformations coincide precisely with the transformations (3.33) derived in Sect. 3.2 provided the following identities take place,

$$
\begin{aligned}
2 \epsilon_{1}^{g} & =\bar{\epsilon}_{1}-\epsilon_{1}, \quad 2 \epsilon_{2}^{g}=\bar{\epsilon}_{2}-\epsilon_{2}, \\
\frac{1}{2} \epsilon_{+}^{g} & =\epsilon_{-}, \quad-\frac{1}{2} \epsilon_{-}^{g}=\bar{\epsilon}_{+}
\end{aligned}
$$

The PB algebra of the charges $Q_{ \pm}, Q_{1}, Q_{2}$ can be evaluated with help of eqns. (3.42), (3.43) and (3.45) yielding the following deformed structure

$$
\begin{aligned}
\left\{Q_{1}, Q_{ \pm}\right\} & = \pm Q_{ \pm} \\
\left\{Q_{2}, Q_{ \pm}\right\} & =0 \\
\left\{Q_{+}, Q_{-}\right\} & =-\left(\frac{2 \pi}{k}\right)^{2} \int \partial_{x} e^{-R_{1}} d x=2 \kappa\left(\frac{2 \pi}{k}\right)^{2} \sinh \left(Q_{1}-\frac{1}{2} Q_{2}\right)
\end{aligned}
$$

where $\kappa=\exp \left(\frac{1}{2}\left(R_{1}(\infty)+R_{1}(-\infty)\right)\right)$. Note that $\kappa$ is a constant operator commuting with all the other generators. Finally one can verify the invariance of the gauged IM (2.25) under 
the above nonlocal transformations by calculating the PB's of the charges $Q_{ \pm}, Q_{i}$ with the hamiltonian of the model

$$
\begin{aligned}
H & =\int d x\left(\left(1+\psi_{2} \chi_{2}\right) \Pi_{\chi_{2}} \Pi_{\psi_{2}}+\frac{1}{2} \psi_{2} \chi_{1} \Pi_{\chi_{1}} \Pi_{\psi_{2}}+\left(1+\psi_{1} \chi_{1}+\psi_{2} \chi_{2}\right) \Pi_{\chi_{1}} \Pi_{\psi_{1}}\right. \\
& \left.+\frac{1}{2} \psi_{1} \chi_{2} \Pi_{\chi_{2}} \Pi_{\psi_{1}}+\psi_{1}^{\prime} \Pi_{\psi_{1}}+\psi_{2}^{\prime} \Pi_{\psi_{2}}-\chi_{1}^{\prime} \Pi_{\chi_{1}}-\chi_{2}^{\prime} \Pi_{\chi_{2}}+V\right)
\end{aligned}
$$

After a tedious but straightforward calculations we find that

$$
\left\{Q_{ \pm}, H\right\}=0, \quad\left\{Q_{i}, H\right\}=0
$$

Hence the IM in consideration is invariant under the algebra (3.48), which after certain rescaling of the generators (see for example ref. [26]) can be identified with the $q$-deformed $S L(2, R) \otimes U(1) \mathrm{PB}$ algebra.

\section{Dressing Transformations and Vertex Operators}

As it is well known [6], [25] the dressing transformation and the vertex operators method represents a powerfull tool for the construction of solitons solutions for the affine Toda models. Let us consider two arbitrary solutions $B_{s} \in \hat{G}_{0}, s=1,2$ of eqns. (2.7) written for the case of $A_{2}^{(1)}$ extended by $d$ and the central term $c$, i.e.

$$
B_{s}=g_{0 s} e^{\nu_{s} c+\eta_{s} d}
$$

The corresponding Lax (L-S) connections $(2.27) \mathcal{A}(s)=\mathcal{A}\left(B_{s}\right), \overline{\mathcal{A}}(s)=\overline{\mathcal{A}}\left(B_{s}\right)$ are related by gauge (dressing) transformations $\theta_{-,+}=\exp \mathcal{G}_{<,>}$,

$$
\mathcal{A}_{\mu}(2)=\theta_{ \pm} \mathcal{A}_{\mu}(1) \theta_{ \pm}^{-1}+\left(\partial_{\mu} \theta_{ \pm}\right) \theta_{ \pm}^{-1}
$$

They leave invariant the equations of motion (2.7) as well as the auxiliar linear problem, i.e. the pure gauge $\mathcal{A}_{\mu}$ defined in terms of the monodromy matrix $T\left(B_{s}\right)$,

$$
\left(\partial_{\mu}-\mathcal{A}\left(B_{s}\right)_{\mu}\right) T_{s}\left(B_{s}\right)=0
$$

The consistency of equations (4.50) and (4.51) imply the following relations

$$
T_{2}=\theta_{ \pm} T_{1}, \text { i.e. } \theta_{+} T_{1}=\theta_{-} T_{1} g^{(1)}
$$

where $g^{(1)} \in \hat{G}$ is an arbitrary constant element of the corresponding affine group. Suppose $T_{1}=T_{0}\left(B_{v a c}\right)$ is the vacuum solution,

$$
\begin{aligned}
B_{v a c} \epsilon_{-} B_{v a c}^{-1} & =\epsilon_{-}, \quad \bar{\partial} B_{v a c} B_{v a c}^{-1}=\mu^{2} z c \\
\mathcal{A}_{v a c} & =-\epsilon_{-}, \quad \overline{\mathcal{A}}_{v a c}=\epsilon_{+}+\mu^{2} z c
\end{aligned}
$$

and $T_{0}=\exp \left(-z \epsilon_{-}\right) \exp \left(\bar{z} \epsilon_{+}\right)$as one can easily check by using the fact that $\left[\epsilon_{+}, \epsilon_{-}\right]=\mu^{2} c$. According to eqns. (4.50) and (4.52), every solution $T_{2}=T(B)$ can be obtained from the 
vacuum configuration (4.53) by an appropriate gauge transformation $\theta_{ \pm}$. In fact, eqns. (4.50) with $\mathcal{A}_{v a c}$ and $\overline{\mathcal{A}}_{v a c}$ as in eqn. (4.53) and

$$
\mathcal{A}(B)=-B \epsilon_{-} B^{-1}, \quad \overline{\mathcal{A}}(B)=\epsilon_{+}+\bar{\partial} B B^{-1}
$$

allows to derive $\theta_{ \pm}$as functionals of $B$, i.e. $\theta_{ \pm}=\theta_{ \pm}(B)$. We next apply eqns. (4.52),

$$
\theta_{-}^{-1} \theta_{+}=T_{v a c} g^{(1)} T_{v a c}^{-1}
$$

in order to obtain a non trivial field configuration $B$ in terms of $g^{(1)} \in G$ and certain highest weight (h.w.) representation of the algebra $A_{2}^{(1)}$ as we shall see below. The first step consists in substituting $\mathcal{A}_{v a c}, \overline{\mathcal{A}}_{\text {vac }}$ and $\mathcal{A}(B), \overline{\mathcal{A}}(B)$ in eqn. (4.50) and then solving it grade by grade remembering that $\theta_{ \pm}$may be decomposed in the form of infinite products

$$
\theta_{-}=e^{t(0)} e^{t(-1)} \cdots, \quad \theta_{+}=e^{v(0)} e^{v(1)} \ldots
$$

where $t(-k)$ and $v(k), k=1,2, \cdots$ denote linear combinations of grade $p=\mp k$ generators. For grade zero we find

$$
t(0)=H(\bar{z}), \quad e^{v(0)}=B e^{G(z)-\mu^{2} z \bar{z} c}
$$

where the arbitrary functions $H(\bar{z}), G(z) \in \mathcal{G}_{0}^{0}$ and are fixed to zero due to the subsidiary constraints (3.34), (3.35), i.e., $H(\bar{z})=G(z)=0$. The equations for $v(1), t(-1)$ appears to be of the form

$$
B^{-1} \partial B-\mu^{2} \bar{z} c=\left[v(1), \epsilon_{-}\right], \quad \bar{\partial} B B^{-1}=\left[t(-1), \epsilon_{+}\right]+\mu^{2} z c
$$

The next step is to consider certain matrix elements (taken for the h.w. representation $\mid \lambda_{l}>$ ) of eqn. (4.54). Since $v(i) \mid \lambda_{l}>=0$ and $\left\langle\lambda_{l}\right| t(-i)=0, i>0$, we conclude that

$$
<\lambda_{l}|B| \lambda_{l}>e^{-\mu^{2} z \bar{z}}=<\lambda_{l}\left|T_{0} g^{(1)} T_{0}^{-1}\right| \lambda_{l}>
$$

Taking into account the explicit parametrization of the zero grade subgroup element $B=$ nam (2.8) in terms of the fields, $\nu, R_{i}, \psi_{a}, \chi_{a}$ and choosing specific matrix elements we derive their explicit space-time dependence,

$$
\begin{aligned}
\tau_{0} \equiv e^{\nu-\mu^{2} z \bar{z}} & =<\lambda_{0}\left|T_{0} g^{(1)} T_{0}^{-1}\right| \lambda_{0}> \\
\tau_{1} \equiv e^{\frac{1}{3}\left(2 R_{1}+R_{2}\right)+\nu-\mu^{2} z \bar{z}}= & <\lambda_{1}\left|T_{0} g^{(1)} T_{0}^{-1}\right| \lambda_{1}> \\
\tau_{2} \equiv e^{\frac{1}{3}\left(R_{1}+2 R_{2}\right)+\nu-\mu^{2} z \bar{z}}= & <\lambda_{2}\left|T_{0} g^{(1)} T_{0}^{-1}\right| \lambda_{2}> \\
\tau_{\psi_{3}} \equiv e^{\frac{1}{3}\left(2 R_{1}+R_{2}\right)+\nu-\mu^{2} z \bar{z}} \tilde{\psi}_{3}= & <\lambda_{1}\left|T_{0} g^{(1)} T_{0}^{-1} E_{-\alpha_{1}-\alpha_{2}}^{(0)}\right| \lambda_{1}>, \\
\tau_{\chi_{3}} \equiv e^{\frac{1}{3}\left(2 R_{1}+R_{2}\right)+\nu-\mu^{2} z \bar{z}} \tilde{\chi}_{3}= & <\lambda_{1}\left|E_{\alpha_{1}+\alpha_{2}}^{(0)} T_{0} g^{(1)} T_{0}^{-1}\right| \lambda_{1}>, \\
\tau_{\psi_{2}} \equiv e^{\frac{1}{3}\left(R_{1}+2 R_{2}\right)+\nu-\mu^{2} z \bar{z}} \tilde{\psi}_{2}= & <\lambda_{2}\left|T_{0} g^{(1)} T_{0}^{-1} E_{-\alpha_{2}}^{(0)}\right| \lambda_{2}> \\
\tau_{\chi_{2}} \equiv e^{\frac{1}{3}\left(R_{1}+2 R_{2}\right)+\nu-\mu^{2} z \bar{z}} \tilde{\chi}_{2}= & <\lambda_{2}\left|E_{\alpha_{2}}^{(0)} T_{0} g^{(1)} T_{0}^{-1}\right| \lambda_{2}> \\
\tau_{\psi_{1}} \equiv e^{\frac{1}{3}\left(2 R_{1}+R_{2}\right)+\nu-\mu^{2} z \bar{z}} \tilde{\psi}_{1}= & <\lambda_{1}\left|T_{0} g^{(1)} T_{0}^{-1} E_{-\alpha_{1}}^{(0)}\right| \lambda_{1}> \\
\tau_{\chi_{1}} \equiv e^{\frac{1}{3}\left(2 R_{1}+R_{2}\right)+\nu-\mu^{2} z \bar{z}} \tilde{\chi}_{1}= & <\lambda_{1}\left|E_{\alpha_{1}}^{(0)} T_{0} g^{(1)} T_{0}^{-1}\right| \lambda_{1}>
\end{aligned}
$$


In order to make the construction of the solution (4.56) complete it remains to specify the constant affine group element $g^{(1)}$, which encodes the information (including topological properties) about the N-soliton structure of eqns. (2.7).

Since $\epsilon_{ \pm}$form a Heisenberg subalgebra, $\left[\epsilon_{+}, \epsilon_{-}\right]=\mu^{2} c$ and we have to calculate the matrix elements ( $\tau$-functions ), say

$$
<\lambda_{0}\left|e^{-z \epsilon_{-}} e^{\bar{z} \epsilon_{+}} g^{(1)} e^{-\bar{z} \epsilon_{+}} e^{z \epsilon_{-}}\right| \lambda_{0}>,
$$

it is instructive to introduce the eigenvectors $(F(\gamma))$ of $\epsilon_{ \pm}$, i.e.,

$$
\left[\epsilon^{ \pm}, F(\gamma)\right]=f^{ \pm}(\gamma) F(\gamma)
$$

Following ref. [24] (see also [11]) we find four non trivial types of eigenvectors

$$
\begin{aligned}
& F_{ \pm}(\gamma)=\sum_{n \in Z}\left(E_{ \pm \alpha_{2}}^{(n)}+E_{ \pm\left(\alpha_{1}+\alpha_{2}\right)}^{(n)}\right) \gamma^{-n} \\
& \tilde{F}_{ \pm}(\gamma)=\sum_{n \in Z}\left(E_{ \pm \alpha_{2}}^{(n)}-E_{ \pm\left(\alpha_{1}+\alpha_{2}\right)}^{(n)}\right) \gamma^{-n}
\end{aligned}
$$

as well as the trivial eigenvectors

$$
F_{ \pm}^{0}(\gamma)=\sum_{n \in Z} E_{ \pm \alpha_{1}}^{(n)} \gamma^{-n}
$$

Their eigenvalues are given by

$$
\begin{aligned}
& {\left[\epsilon_{+}, F_{ \pm}(\gamma)\right]= \pm \mu \gamma F_{ \pm}(\gamma), \quad\left[\epsilon_{+}, \tilde{F}_{ \pm}(\gamma)\right]= \pm \mu \gamma \tilde{F}_{ \pm}(\gamma), \quad\left[\epsilon_{+}, F_{ \pm}^{0}(\gamma)\right]=0} \\
& {\left[\epsilon_{-}, F_{ \pm}(\gamma)\right]= \pm \mu \gamma^{-1} F_{ \pm}(\gamma), \quad\left[\epsilon_{-}, \tilde{F}_{ \pm}(\gamma)\right]= \pm \mu \gamma^{-1} \tilde{F}_{ \pm}(\gamma), \quad\left[\epsilon_{-}, F_{ \pm}^{0}(\gamma)\right]=0}
\end{aligned}
$$

Notice that $F_{ \pm}(\gamma), \tilde{F}_{ \pm}(\gamma)$ and $F_{ \pm}^{0}(\gamma)$ together with

$$
\lambda_{i} \cdot H(\gamma)=\sum_{n \in Z} \lambda_{i} \cdot H^{(n)} \gamma^{-n}, \quad i=1,2
$$

form a new basis for the affine $A_{2}^{(1)}$ algebra. In this basis, we define the affine group element $g^{(1)}$ as

$$
g^{(1)}=\prod_{a} e^{d_{a} F_{a}(\gamma)}, \quad F_{a}(\gamma)=\left\{F_{ \pm}, \tilde{F}_{ \pm}, \lambda_{i} \cdot H(\gamma), \quad i=1,2, \quad F_{ \pm}^{0}(\gamma)\right\}
$$

with the property

$$
\begin{aligned}
T_{0} g^{(1)} T_{0}^{-1} & =\exp \left(\sum_{a} d_{a} \rho_{a}(\gamma) F_{a}(\gamma)\right)=\prod_{a}\left(1+d_{a} \rho_{a}(\gamma) F_{a}(\gamma)\right) \\
\rho_{a}(\gamma) & =\exp \left(-z f_{a}^{-}(\gamma)+\bar{z} f_{a}^{+}(\gamma)\right)
\end{aligned}
$$

The use of this basis drastically simplify the calculation of the $\tau$-functions (4.56). Notice that in the above formula each $F_{a}^{2}=0$, but the mixed terms $F_{a} F_{b}$ do contribute [24]. 


\section{Two-Vertex Soliton Solutions}

An important question concerns the specific choice of the form of $g^{(1)}$ that leads to different species of neutral and charged solitons and breathers. As in the case of complex sine-Gordon model the 1-soliton solutions can be constructed in terms of two vertex operators, i.e., $g^{(1)}$ chosen in one of the following forms:

$$
\begin{gathered}
g^{(1)}\left(\gamma_{1}, \gamma_{2}\right)=e^{d_{1} F_{+}\left(\gamma_{1}\right)} e^{d_{2} F_{-}\left(\gamma_{2}\right)} \\
\tilde{g}^{(1)}\left(\gamma_{1}, \gamma_{2}\right)=e^{\tilde{d}_{1} \tilde{F}_{+}\left(\gamma_{1}\right)} e^{\tilde{d}_{2} \tilde{F}_{-}\left(\gamma_{2}\right)} \\
g_{01}^{(1)}\left(\gamma_{1}, \gamma_{2}\right)=e^{d_{01} F_{+}\left(\gamma_{1}\right)} e^{\tilde{d}_{01} \tilde{F}_{-}\left(\gamma_{2}\right)} \\
g_{02}^{(1)}\left(\gamma_{1}, \gamma_{2}\right)=e^{\tilde{d}_{02} \tilde{F}_{+}\left(\gamma_{1}\right)} e^{d_{02} F_{-}\left(\gamma_{2}\right)}
\end{gathered}
$$

For the case given by eqn. (5.63) according to (4.62) we find for the $\tau$-functions (4.56),

$$
\begin{aligned}
\tau_{0} & =1+2 d_{1} d_{2} \rho_{1}\left(\gamma_{1}\right) \rho_{2}\left(\gamma_{2}\right) \frac{\gamma_{1} \gamma_{2}}{\left(\gamma_{1}-\gamma_{2}\right)^{2}}, \\
\tau_{1} & =1+d_{1} d_{2} \rho_{1}\left(\gamma_{1}\right) \rho_{2}\left(\gamma_{2}\right) \frac{\gamma_{1}\left(\gamma_{1}+\gamma_{2}\right)}{\left(\gamma_{1}-\gamma_{2}\right)^{2}}, \\
\tau_{2} & =1+2 d_{1} d_{2} \rho_{1}\left(\gamma_{1}\right) \rho_{2}\left(\gamma_{2}\right) \frac{\gamma_{1}^{2}}{\left(\gamma_{1}-\gamma_{2}\right)^{2}}, \\
\tau_{\psi_{3}} & =\tau_{\psi_{2}}=d_{1} \rho_{1}\left(\gamma_{1}\right), \quad \tau_{\chi_{3}}=\tau_{\chi_{2}}=d_{2} \rho_{2}\left(\gamma_{2}\right), \\
\tau_{\psi_{1}} & =\tau_{\chi_{1}}=d_{1} d_{2} \rho_{1}\left(\gamma_{1}\right) \rho_{2}\left(\gamma_{2}\right) \frac{\gamma_{1}}{\gamma_{1}-\gamma_{2}},
\end{aligned}
$$

where $\rho_{1}\left(\gamma_{1}\right)=\exp \left(-\frac{z}{\gamma_{1}}+\bar{z} \gamma_{1}\right)$ and $\rho_{2}\left(\gamma_{2}\right)=\exp \left(\frac{z}{\gamma_{2}}-\bar{z} \gamma_{2}\right)$. In order to ensure the reality (and positivity) of the energy of the solution, we require that the product $\rho_{1}\left(\gamma_{1}\right) \rho_{2}\left(\gamma_{2}\right)$ to be real. This leads to the following parametrization for $\gamma_{i}$ :

$$
\gamma_{1}=-e^{b-i \alpha}, \quad \gamma_{2}=e^{b+i \alpha}, \quad b, \alpha \in R
$$

and for $\rho_{i}\left(\gamma_{i}\right)$ we obtain,

$$
\begin{aligned}
\rho_{1}=e^{F+i G}, \quad \rho_{2}=e^{F-i G}, \quad z & =\frac{1}{2}(x+t), \quad \bar{z}=\frac{1}{2}(t-x) \\
F=\mu \cos (\alpha)[-t \operatorname{sh}(b)+x \operatorname{ch}(b)], \quad G & =\mu \sin (\alpha)[t \operatorname{ch}(b)-x \operatorname{sh}(b)]
\end{aligned}
$$

It is convenient to choose the arbitrary complex constants $d_{1}$ and $d_{2}$ in the form:

$$
d_{1}=\frac{\gamma_{1}-\gamma_{2}}{\sqrt{2 \gamma_{1} \gamma_{2}}} e^{i \theta-\mu Y \cos (\alpha) \operatorname{ch}(b)}, \quad d_{2}=\frac{\gamma_{1}-\gamma_{2}}{\sqrt{2 \gamma_{1} \gamma_{2}}} e^{-i \theta-\mu Y \cos (\alpha) \operatorname{ch}(b)}
$$


where $\theta$ and $Y$ are new arbitrary real constants. Then the 1-soliton solutions corresponding to two vertex $g^{(1)}(5.63)$ takes the following simple form:

$$
\begin{aligned}
e^{\nu-\mu^{2} z \bar{z}} & =1+e^{2 \tilde{F}} \\
e^{\frac{1}{3}\left(2 R_{1}+R_{2}\right)} & =\frac{e^{-\tilde{F}}+\frac{1}{2}\left(1-\Gamma_{1}\right) e^{\tilde{F}}}{e^{\tilde{F}}+e^{-\tilde{F}}}, \quad \Gamma_{1}=e^{-2 i \alpha} \\
e^{\frac{1}{3}\left(R_{1}+2 R_{2}\right)} & =\frac{e^{-\tilde{F}}-\Gamma_{1} e^{\tilde{F}}}{e^{\tilde{F}}+e^{-\tilde{F}}}, \quad \Gamma_{2}=d_{1} e^{-i \theta+\mu Y \cos (\alpha) c h(b)}=\frac{\gamma_{1}-\gamma_{2}}{\sqrt{2 \gamma_{1} \gamma_{2}}} \\
\psi_{1} & =\frac{\Gamma_{2} e^{i(G+\theta)}}{\left(e^{\tilde{F}}+e^{-\tilde{F}}\right)}\left(\frac{e^{-\tilde{F}}-\Gamma_{1} e^{\tilde{F}}}{e^{-\tilde{F}}+\frac{1}{2}\left(1-\Gamma_{1}\right) e^{\tilde{F}}}\right)^{\frac{1}{2}} \\
\chi_{1} & =\frac{\Gamma_{2} e^{-i(G+\theta)}}{\left(e^{\tilde{F}}+e^{-\tilde{F}}\right)}\left(\frac{e^{-\tilde{F}}-\Gamma_{1} e^{\tilde{F}}}{e^{-\tilde{F}}+\frac{1}{2}\left(1-\Gamma_{1}\right) e^{\tilde{F}}}\right)^{\frac{1}{2}} \\
\psi_{2} & =\frac{\Gamma_{2} e^{i(G+\theta)}}{\left(e^{\tilde{F}}+e^{-\tilde{F}}\right)}\left(\frac{e^{-\tilde{F}}+e^{\tilde{F}}}{e^{-\tilde{F}}+\frac{1}{2}\left(1-\Gamma_{1}\right) e^{\tilde{F}}}\right)^{\frac{1}{2}} \\
\chi_{2} & =\frac{\Gamma_{2} e^{-i(G+\theta)}}{\left(e^{\tilde{F}}+e^{-\tilde{F}}\right)}\left(\frac{e^{-\tilde{F}}+e^{\tilde{F}}}{e^{-\tilde{F}}+\frac{1}{2}\left(1-\Gamma_{1}\right) e^{\tilde{F}}}\right)^{\frac{1}{2}}
\end{aligned}
$$

where $\tilde{F}(t, x)=F(t, x-Y)$. The nonlocal fields $\tilde{\psi}_{1}$ and $\tilde{\chi}_{1}$ (whose asymptotics are important for determining the charges $Q_{ \pm}$) are given by:

$$
\tilde{\psi}_{1}=\tilde{\chi}_{1}=-\frac{\left(1+\Gamma_{1}\right) e^{2 \tilde{F}}}{2\left(1+\frac{1}{2}\left(1-\Gamma_{1}\right) e^{2 \tilde{F}}\right)}
$$

As it is well known [6], [7], [10] the energy of such solution is related to the asymptotics of $\ln \tau_{0}$, i.e.,

$$
M=E(b=0)=\int_{-\infty}^{\infty} d x T_{00}=-\frac{2}{\beta^{2}} \int_{-\infty}^{\infty} d x \partial_{x} \ln \tau_{0}=\frac{4 \mu}{\beta^{2}} \cos (\alpha), \quad \beta^{2}=\frac{2 \pi}{k}
$$

in the rest frame $b=0$. The corresponding Noether charges $Q_{1}$ and $Q_{2}$ (see eqns. (3.38)) are defined by the asymptotics of the nonlocal fields $R_{i}$

$$
Q_{1}=-i(\alpha+\pi+i \ln (\cos (\alpha))), \quad Q_{2}=-i(\alpha+\pi+i \ln (2 \cos (\alpha)))
$$

Similarly, for the charges $Q_{ \pm}$we find

$$
Q_{+}=Q_{-}=\tilde{\psi}_{1}(\infty)-\tilde{\psi}_{1}(-\infty)=\frac{\Gamma_{1}+1}{\Gamma_{1}-1}=i \operatorname{cotg}(\alpha)
$$

Therefore the spectrum of the above 1 -soliton solutions, i.e., $M, Q_{1}, Q_{2}$ and $Q_{ \pm}$is determined by the real constant $\alpha$ only. 
The case when $g^{(1)}$ is taken in the form (5.64) is quite similar to the considered above. The only difference is that now we have

$$
\tau_{\psi_{3}}=-\tau_{\psi_{2}}, \quad \tau_{\chi_{3}}=-\tau_{\chi_{2}}, \quad \tau_{\psi_{1}}=\tau_{\chi_{1}}=-\tilde{d}_{1} \tilde{d}_{2} \rho_{1} \rho_{2} \frac{\gamma_{1}}{\gamma_{1}-\gamma_{2}}
$$

and all the other tau-functions remain unchanged. As a consequence, the mass $\tilde{M}$ and charges $\tilde{Q}_{1}, \tilde{Q}_{2}$ are the same and $\tilde{Q}_{ \pm}=-Q_{ \pm}$. The cases (5.65) and (5.66) are quite different. We find that

$$
\begin{aligned}
\tau_{0} & =\tau_{2}=1, \quad \tau_{1}=1-d_{1} \tilde{d}_{2} \rho_{1}\left(\gamma_{1}\right) \rho_{2}\left(\gamma_{2}\right) \frac{\gamma_{1}}{\gamma_{1}-\gamma_{2}} \\
\tau_{\psi_{1}} & =-\tau_{\chi_{1}}=d_{1} \tilde{d}_{2} \rho_{1}\left(\gamma_{1}\right) \rho_{2}\left(\gamma_{2}\right) \frac{\gamma_{1}}{\gamma_{1}-\gamma_{2}} \\
\tau_{\psi_{3}} & =-\tau_{\psi_{2}}=d_{1} \rho_{1}\left(\gamma_{1}\right), \quad \tau_{\chi_{3}}=-\tau_{\chi_{2}}=-\tilde{d}_{2} \rho_{2}\left(\gamma_{2}\right)
\end{aligned}
$$

Such solution has vanishing mass and charge $Q_{2}=0$.

\section{Concluding Remarks}

Our analysis of the symmetries and 1-soliton solutions of the IM (2.25) leaves few interesting open problems:

- How to construct more general 1-soliton solutions whose spectrum, $M, Q_{1}, Q_{2}$ and $Q_{ \pm}$ is parametrized by four real parameters instead of one $\alpha$ as in eqn. (5.68)

- What are the symmetry properties of the 1-solitons (5.68), i.e. to recognize the representations of the $q$-deformed algebra (3.48) to which these solitons belong to.

- About the topological stability of these solitons and of the related strong coupling particles of the IM (2.25)

The complete structure of the solitons (and particles) spectrum of such IM ideed require to answer the above questions.

Acknowledgments One of us (ICC) would like to thank P. Teotonio Sobrinho for discussions. We thank CNPq and Fapesp for support.

\section{References}

[1] V. Fateev and A. Zamolodchikov, Int. J. Mod. Phys. A5 (1990) 1025

[2] P. Christe and G. Mussardo, Nucl. Phys. B330 (1990) 465 ; Int. J. Mod. Phys. A5 (1990) 4581

[3] G. Sotkov and C.J. Zhu, Phys. Lett. 229B (1989) 391 
[4] G.Sotkov and G. Mussardo, "S-matrix bootstrap and minimal integrable models", in Recent Developments in Conformal Field Theory, Trieste Conference, October 1989, Eds. J-B. Zuber et. all. World Scientific, p. 231-270.

[5] T. Hollowood, Nucl. Phys. 384, (1992), 523

[6] D. Olive, N. Turok and J. Underwood, Nucl. Phys. B409 (1993) 509 ; Nucl. Phys. B401 (1993) 663

[7] H. Aratyn, C.P. Constantinidis, L.A. Ferreira, J.F. Gomes and A.H. Zimerman,Nucl. Phys. B406 (1993) 727

[8] I. Affleck, "Quantum Theory Methods and Quantum Critical Phenomena", in Field Strings and Critical Phenomena, Les Houches XLIX, (1998); I. Affleck, Nucl. Phys. B257 (1985) 397; I. Affleck and F.D.M. Haldane, Phys. Rev. B36 (1987) 5291

[9] J.F. Gomes, E.P. Gueuvoghlanian, G.M. Sotkov and A.H. Zimerman, Nucl. Phys. B606 (2001) 441, hep-th/0007169

[10] J.F. Gomes, E.P. Gueuvoghlanian, G.M. Sotkov and A.H. Zimerman, Nucl. Phys. B598(2001) 615, hepth/0011187,

[11] I. Cabrera-Carnero, J.F. Gomes, G.M. Sotkov and A.H. Zimerman, Nucl. Phys. B634 (2002) 433 , hep-th/0201047

[12] J.F. Gomes, E.P. Gueuvoghlanian, G.M. Sotkov and A.H. Zimerman, JHEP 0207:001,2002, hep-th/0205228

[13] R.F. Dashen, B. Hasslacher and A. Neveau, Phys. Rev. D10 (1974) 4130; Phys. Rev. D11 (1975) 3424; R. Jackiw and G. Woo, Phys. Rev. D12 (1975) 1643

[14] T. Hollowood and N. Dorey,Nucl. Phys. B440 (1995) 215, hep-th/9410140

[15] J.F. Gomes, E.P. Gueuvoghlanian, G.M. Sotkov and A.H. Zimerman, Ann. of Phys. 289 (2001) 232, hep-th/0007116, see also "Torsionless T Selfdual Affine NA Toda Models",Proc. of the IV International Wigner Symposium, Istambul, Ed. M. Arik et. al., hep-th/0002173; J.F. Gomes, G.M. Sotkov and A.H. Zimerman, Proc. of Workshop on Integrable Theories, Solitons and Duality, Sao Paulo, Brazil, JHEP, unesp2002/045, hep-th/0212046

[16] V. Fateev, Nucl. Phys. B479 (1996) 594 ; Nucl. Phys. B473 (1996) 509

[17] L.A. Ferreira, J.L. Miramontes, J. Sanchez Guillen, Nucl. Phys. B449 (1995) 631; J. Math. Phys. 38 (1997) 882

[18] A. N. Leznov, M. V. Saveliev, Group Theoretical Methods for Integration of Nonlinear Dynamical Systems, Progress in Physics, Vol. 15 (1992), Birkhauser Verlag, Berlin; A. N. Leznov and M. V. Saveliev, Commun. Math. Phys. 89 (1983) 59 
[19] J.F. Gomes, G.M. Sotkov and A.H. Zimerman, J. Physics A37 (2004) 4629, hepth/0402091

[20] A. Fordy and P. Kulish, Commun. Math. Phys. 89 (1983) 427

[21] C.R. Fernandez-Pousa, M.V. Gallas, T.J. Hollowood and J.L. Miramontes, Nucl. Phys. B484 (1997) 609, Nucl. Phys. B499 (1997) 673

[22] H. Aratyn, J.F. Gomes and A.H. Zimerman, J. Math. Phys. 36,(1995),3419, hepth/9408104

[23] F. Lund, Ann. of Phys. 415 (1978) 251;

F. Lund and T. Regge, Phys. Rev. D14 (1976) 1524

[24] H. Aratyn, L.A. Ferreira, J.F. Gomes and A.H. Zimerman, J. Phys. A31, (1998) 94839492, solv-int/9709004

[25] O. Babelon and D. Bernard, Int. J. Mod. Phys. A8 (1993) 507

[26] D. Bernard and A. Leclair, Commun. Math. Phys. 142 (1991) 99 